\title{
Problemas en el adolescente, mindfulness y rendimiento escolar en estudiantes de secundaria. Estudio preliminar
}

\section{Problems in Adolescents, Mindfulness and School Performance in High School Students. Preliminary Study}

\author{
Alberto Mercader Rovira \\ Universidad de Lleida, Lleida, España \\ ORCID: http://orcid.org/0000-0002-0461-657X
}

Recibido 05-08-19 Revisado 27-10-19 Aprobado 28-11-19 En línea 02-12-19

Correspondencia

Email: amercaderr@gmail.com
Citar como:

Mercader, A. (2020). Problemas en el adolescente,
mindfulness y rendimiento escolar en estudiantes de
secundaria. Estudio preliminar. Propósitos $y$
$\begin{array}{lll}\text { Representaciones, } & 8(1), & \text { e372. }\end{array}$ doi:
http://dx.doi.org/10.20511/pyr2020.v8n1.372

Mercader, A. (2020). Problemas en el adolescente escolar en estudiantes de http://dx.doi.org/10.20511/pyr2020.v8n1.372 


\section{Resumen}

En este trabajo se discute la importancia de la educación emocional para reducir los problemas del adolescente y se destaca la competencia emocional y auto regulación como elementos básicos de la educación por competencias, a raíz de las investigaciones vinculadas al papel de las Funciones Ejecutivas. Además, se presentan algunos resultados de programas y terapias con Mindfulness o atención consciente al momento presente en niños y adolescentes y se constata la poca presencia de investigaciones, aún, en Perú. Se presentan los resultados de un estudio ex post facto con una muestra $(\mathrm{N}=76)$ de alumnos de Secundaria de la IE Jorge Basadre Grohmann de Trujillo, Perú, que describió los problemas de los adolescentes y los comparó con la capacidad de atención consciente y el rendimiento académico. Se observó que los alumnos presentaban significación clínica en problemas de autoestima $(25,4 \%)$ y depresión $(11,8 \%)$ y que hubo relaciones bajas entre la capacidad de ser consciente al momento presente con ansiedad ( $\rho=-, 349$, $p, 005)$, depresión $(\rho=-, 473, p, 000)$, problemas interpersonales $(\rho=-, 309, p, 014)$, incertidumbre sobre el futuro $(\rho=-, 421, p, 001)$ y riesgo psicosocial $(\rho=-, 409, p, 001)$. No se detectaron relaciones significativas entre los problemas del adolescente y las notas académicas, aunque se observaron asociaciones entre las calificaciones. El artículo pretende aportar un grano más de arena para el estudio de los problemas emocionales de los adolescentes peruanos con el objetivo de impulsar políticas de educación basadas en las emociones, una temática en la que aún hay campo por recorrer.

Palabras clave: Proceso de aprendizaje; Rendimiento escolar; Psicología del adolescente; Salud mental; Pensamiento.

\section{Summary}

This paper discusses the importance of emotional education to reduce adolescent problems and emphasizes emotional competence and self-regulation as basic elements of competency education in the school context, based on research in recent decades, especially linked to the role of Executive Functions. In addition, some results of programs and therapies with Mindfulness or conscious attention to the present moment in children and adolescents are presented and the little presence of research is observed, even in Peru. The results of an ex post facto study are presented with a sample $(\mathrm{N}=76)$ of IE High School students Jorge Basadre Grohmann from Trujillo, Peru, where is described the problems in adolescents and compared them with the capacity for conscious attention and academic performance. We noted that the students presented clinical significance in problems of self-esteem (25.4\%) and depression (11.8\%) and that there was few relationship between the ability to be aware at the present moment with anxiety $(\rho=-, 349, p, 005)$, depression $(\rho=-473, p, 000)$, interpersonal problems $(\rho=-, 309, p, 014)$, uncertainty about the future $(\rho=, 421, p, 001)$ and psychosocial risk $(\rho=-, 409, p, 001)$. No significant relationships were detected between adolescent problems and academic grades, although associations between grades were observed. The article aims to make another contribution for the study of the emotional problems in Peruvian adolescents and to promote the development of education policies based on emotions, a theme in which there is still a long way to go.

Keywords: Learning Processes; Academic achievement; Adolescent psychology; Mental Health; Thought Process.

\section{Introducción}

La adolescencia es el periodo de crecimiento y desarrollo que se sitúa entre la niñez y la adultez, entre los 10 y los 19 años. La OMS considera la adolescencia una etapa marcada por los procesos biológicos y muy condicionada por las experiencias y el contexto social, que puede tener un efecto 
"considerable" (OMS, s.f.) y destaca la necesidad de desarrollar acciones de promoción de la salud en niños y adolescentes, acorde con las políticas de estrategia global en la salud (Southall et al., 2000).

El concepto de la adolescencia es relativamente nuevo. La revolución industrial, a mediados del S.XVIII, supuso un cambio en la consideración del niño adulto que existía hasta entonces. Ariès (1987) nos recuerda que antes que empezase la industrialización el niño que abandonaba sus pañales era entrenado en los talleres de artesanía para que se integrara como un adulto en la sociedad. Pasaba de niño a adulto. A partir del siglo XX es cuando se comienza a estudiar al adolescente. Los pioneros en el estudio (Stanley Hall con su obra Adolescence publicada en 1904 y su conocido acuño de la adolescencia como un tiempo de "storm and stress", Margaret Mead (1993) con su estudio publicado en 1939 sobre el sexo y la cultura o Kurl Lewin (1939) con su investigación sobre la inestabilidad del ambiente y la influencia en el niño y el adolescente) pusieron las bases para la investigación de este periodo, que sigue en nuestros días desde muy diversas teorías (Lozano, 2014).

El avance en la tecnología médica, sobre todo la capacidad de obtener imágenes de la actividad cerebral, en estas últimas décadas, ha permitido estudiar las relaciones que existen entre la maduración neuropsicológica del cerebro adolescente y su conducta y evidenciar el papel crucial que juegan diversas regiones cerebrales en este proceso. Entre las diversas aportaciones sobre el estudio de la adolescencia destaca la vinculación que el aumento de hormonas esteroideas, los andrógenos en los varones y los estrógenos en las mujeres, tendría en los problemas que conlleva esta etapa vital, aunque no hay consenso en esta temática. Diversas investigaciones relativizan el papel que estas hormonas tienen en las diversas problemáticas que aquejan los jóvenes (Graber \& Books-Gunn, 1996; Spear, 2007).

Otro de los descubrimientos que ha contribuido al entendimiento de cómo procesa el cerebro el aprendizaje es la lateralización del cerebro. Los estudios han demostrado que el hemisferio derecho cuenta con más áreas de asociación sensorial e intermodal y por tanto tendría un papel más importante en el proceso de adquirir nuevos aprendizajes (Goldberg \& Costa, 1981) aunque el hemisferio izquierdo superaría en capacidad al derecho en la eficacia del procesamiento en base a conocimientos ya codificados (Tirapu-Ustárroz, \& Díaz-Leiva, 2018). Los diferentes estudios sobre el procesamiento cerebral han posibilitado la creación de nuevos constructos sobre su funcionalidad. Uno de ellos es el conocido como Cerebro Social, una red de interconexiones neuronales que estaría localizada anatómicamente entre la corteza prefrontal medial y el surco temporal superior posterior, regiones implicadas en la capacidad de atribuir estados mentales propios y de los demás. Algunos autores relacionarían la redistribución sináptica que se produce a lo largo de la adolescencia en esta región cerebral con los cambios que se suceden en el proceso de maduración de los jóvenes (Blakemore, 2008). Otra de las nuevas definiciones que han aparecido en estos últimos años ha sido el de las Funciones Ejecutivas (FE), vinculadas al lóbulo frontal y concretamente a la corteza prefrontal. De acuerdo con diversos autores, las FE son un conjunto de capacidades que están relacionadas, principalmente, con la organización, el control inhibitorio, la flexibilidad, la memoria de trabajo y la planificación (Flores, Ostrosky \& Lozano 2012; Diamond, 2013). Por ende, la conceptualización de este término ha propiciado otro: el conocido como Control Ejecutivo, que se utiliza para definir la capacidad del individuo de focalizar su atención y pensamientos, anticipándose a las situaciones futuras y planificar acciones en esta dirección (Anderson, 1992). Otros estudios apuntan que las FE también están estrechamente relacionadas con la regulación emocional, por las eferencias que existen entre esta área y la región límbica (Zelazo \& Cunningham, 2007; Shmeichel \& Tang, 2013).

Una de las investigaciones mundialmente conocida que analiza el control ejecutivo y la conducta humana lo realizó el psicólogo austríaco Walter Mischel con el test de la golosina. En ese estudio midió la capacidad de los niños para autocontrolarse ante una golosina bajo la promesa 
de otra posteriormente y describió las correlaciones que existían 14 años después (Mischel, 2015). Los datos que obtuvo fueron que los niños que se habían controlado más ante la golosina tenían mejores puntuaciones en su rendimiento académico, resultados cognitivos y éxito social cuando eran adolescentes que aquellos que no habían podido controlarse, que obtenían peores calificaciones. Además, presentaban imágenes del cerebro diferentes en zonas vinculadas a las adicciones y la obesidad.

Las evidencias sobre la relación del autocontrol en el rendimiento académico han contribuido a la inclusión dentro de los sistemas educativos de las competencias, en las cuales se prioriza la autorregulación como elemento básico del aprendizaje. En Europa, uno de los primeros continentes que apostaron por esta concepción del aprendizaje, se introdujo la educación por competencias a partir de la recomendación del Parlamento Europeo y del Consejo a los Estados miembros de la UE, en qué situaban en 8 las competencias clave para el aprendizaje permanente (dictamen $\mathrm{N}^{\circ} 962,2006$ ). La recomendación insistía en la motivación y confianza como elementos cruciales para el desarrollo de la competencia Aprender a aprender y la capacidad para gestionar la frustración y el estrés como fundamentales en el desempeño de las Competencias Sociales y Cívicas.

El nuevo Currículo Nacional de la Educación Básica de Perú (Ministerio de Educación, 2016), en actual proceso de despliegue en sus tres niveles, sustenta el aprendizaje también por competencias e incluye el concepto Aprender a aprender, aunque no con las mismas palabras utilizadas en Europa. El nuevo currículo nacional sitúa en 31 las competencias que los alumnos deben acreditar en su egreso de la Educación Secundaria. La primera de estas competencias es Construye su identidad, en la cual se trabaja la autorregulación de las emociones. El Ministerio de Educación sitúa como "destacado" este nivel de competencia (Resolución ministerial 1592007, p.2). En el currículo peruano, se destaca también la competencia Convive y participa democráticamente en la búsqueda del bien común, la cual incluye como capacidades el manejo de los conflictos de forma constructiva. En el momento de publicar este documento, el Ministerio de Educación está trabajando en la implantación por fases de las nuevas calificaciones por logros que sustituirán las calificaciones numéricas del 0 al 20, que aún se utilizan de Segundo a Quinto de Educación Secundaria, por las alfabéticas en cuatro niveles implementando así la capacitación del alumno peruano como desarrolla el currículo (Ministerio de Educación, 2019).

\section{Competencia emocional}

Consideramos la emoción como la respuesta química que se crea a partir de una serie de reacciones en la región límbica, integrada por la corteza cingulada, la amígdala y el hipocampo (Sporzon, 2016). Las conductas que tenemos a lo largo del día están estrechamente relacionadas con las interconexiones que se establecen entre el neocórtex frontal y la amígdala, aunque no son las únicas. Las investigaciones sobre la conducta y las emociones cuentan con dos nombres propios muy destacados: Antonio Damasio y David Goleman, que centraron su interés en esta temática. Estos autores plantearon la relación bidireccional entre lo que se consideraba hasta entonces como razón y emoción. Damasio (1996) argumentó, en base a las evidencias científicas que existían hasta entonces, que la facultad de razonamiento es una capacidad vinculada a las reacciones emocionales del individuo destripando así la dualidad cartesiana. Por su parte, Goleman (1995) popularizó el término inteligencia emocional, ya planteada por Salovey y Mayer (1990), como la capacidad para dirigir los pensamientos y acciones y al mismo tiempo gestionar las emociones que se generan. Si tomáramos el símil del interruptor, diríamos que la amígdala es el tejido que enciende esa reacción química de la que hablábamos mientras el córtex frontal sería el encargado de desconectarla (Goleman, 1995). Otros autores (Bisquerra \& Pérez, 2007) han desarrollado este planteamiento postulando las competencias emocionales como la capacidad de movilizar las emociones con un cierto nivel de calidad y eficacia. Independientemente del debate científico que se ha planteado sobre si el constructo inteligencia emocional existe o si es válido o 
no (Davies, Stankov \& Roberts, 1998; Saarni, 2000) parece que existiría un relativo consenso sobre el nuevo término competencia emocional y los beneficios que aporta el desarrollo de estas en el contexto escolar. Hay estudios que relacionan las competencias emocionales con la regulación emocional, la resiliencia o el bienestar subjetivo (Saarni, 2000). Otros han planteado que las competencias emocionales ejercen como mediadoras entre el clima de clase y el rendimiento escolar (López-González \& Oriol-Granado, 2016). Se ha postulado la inteligencia emocional como una capacidad relacionada con la conducta prosocial (Caprara \& Bonino, 2006; Goleman, 2006). En Perú se ha analizado el constructo también en un estudio evidenciando la relación de la inteligencia emocional con la convivencia escolar y el estado de ánimo (Egocheaga, 2017).

\section{Mindfulness}

Uno de los instrumentos que han sido utilizados para reducir el estrés, cultivar la capacidad de estar atento, fomentar la consciencia y aumentar el bienestar subjetivo es el Mindfulness. La palabra Mindfulness (que se traduce como atención o consciencia plena) fue introducida por John Kabat-Zinn, a finales de la década de los años setenta del siglo pasado, a través del Programa de Mindfulness Basado en la Reducción del Estrés, MBRS, en qué proponía prácticas meditativas y atención y consciencia al momento presente a sus pacientes con la intención de mitigar sus síntomas (Kabat-Zinn, 2003). El Mindfulness ancla sus raíces en las prácticas que Buda recomendaba en la población para encontrar una armonía consigo mismo y con la sociedad (Harvey, 2012) aunque abandona toda vinculación religiosa. El Mindfulness propone centrarse en el momento presente y vivir cada momento de una forma activa, aceptando aquello que sucede sin juicios (Vallejo, 2006). Baer, Smith, Hopkins, Krietemeyer y Toney (2006) conceptualizan la capacidad de atención consciente del individuo en cinco facetas: actuar con atención, describir las propias experiencias, no reactividad sino responder adecuadamente, aceptar sin juzgar y observar. Bajo un análisis contextual de los problemas, el Mindfulnesss postula, junto a las terapias de aceptación y compromiso, que las situaciones externas se relacionan con las experiencias internas del individuo y que la eliminación de esas experiencias internas solo hace que el problema se mantenga o aumente el malestar del individuo (Hayes, Strosahl \& Wilson, 1999), lo cual implicaría que la solución está en la capacidad de gestionar el sufrimiento, no en su eliminación. El Mindfulness se ha difundido entre la población mundial adulta en estos últimos años con rapidez después de constatar su efectividad para regular las emociones, optimizar las pautas cognitivas y reducir de la reactividad al estrés. Además, se han reportado mejoras en la respuesta inmunológica, mejoras en el bienestar físico y mejoras en la capacidad de empatía y compasión (Siegel, 2007). Las prácticas de atención o consciencia plena, integradas dentro de las terapias cognitivas-conductuales de tercera generación, cuentan con gran aceptación en Norteamérica donde aparecen ya los primeros estudios sobre su intervención, también, en las fuerzas armadas (Jha et al., 2015; Zanesco, Denkova, Rogers, MacNulty, \& Jha, 2018). En los últimos años, los profesionales de la Psicología han comenzado a aplicar la terapia de la consciencia plena en la población infantil y adolescente, en diversos trastornos mentales sustituyendo las tradicionales terapias cognitivo-conductual.

Desde 2005, el número de estudios e investigaciones sobre esta práctica y el análisis de su eficacia, con niños y adolescentes, se ha ido incrementando (García-Campayo, Demarzo y Modrego, 2017). Un metaanálisis con muestras clínicas informó de su efectividad en la mejora de síntomas psicológicos con prácticas Mindfulness (Zoogman, Goldberg, Hoyt \& Miller, 2015). Otro meta análisis de estudios en el contexto educativo mostró su efectividad en el desempeño cognitivo y relaciones significativas en medidas de estrés, afrontamiento y resiliencia (Zenner, Herrnleben-Kurz \& Walach, 2014). Investigaciones realizadas con estudiantes de Secundaria, la población que se analiza en este estudio, detectaron una reducción significativa del estrés y mejora del bienestar general (Kuyken et al., 2013) o reducciones significativas en la ansiedad estado, ansiedad rasgo y un aumento de velocidad en el procesamiento de la información, inteligencia 
práctica y creatividad (So \& Orme-Johnson, 2001). A pesar que la práctica del Mindfulness comienza a mostrar resultados prometedores en la mejora del funcionamiento global del adolescente, no podemos obviar que los estudios realizados adolecen de limitaciones metodológicas como: el pequeño número de las muestras y escasa representatividad, ensayos sin grupos de control adecuado, escasos estudios cualitativos, heterogeneidad de los programas utilizados, falta de información sobre los detalles del estudio, resultados a corto plazo y dificultades en las medidas de evaluación que limitarían su validez y fiabilidad (García-Campayo et al., 2017). Aun así, los mismos autores indican que la mayoría de los cuestionarios utilizados en los estudios para medir la capacidad de Mindfulness en la población infantil y adolescente cuentan con propiedades psicométricas correctas y que hay camino por recorrer, sobre todo en la adaptación de estos cuestionarios a poblaciones hispanas. En Perú, la investigación sobre el Mindfulness cuenta con pocas referencias. Se han publicado algunos artículos de divulgación en revistas indexadas peruanas (Moscoso \& Lengacher, 2015; Vásquez-Dextre, 2016; Oblitas, et al., 2018), un estudio de traducción de una escala breve de atención consciente (Caycho-Rodríguez, Ventura-León, Martínez-Munive \& Barboza-Palomino, 2019) y se han realizado diversas investigaciones con muestras peruanas: un estudio para analizar la eficacia de un tratamiento que combinaba Mindfulness con aceite esencial (Soto-Vásquez \& Alvarado-García, 2016), una tesis de pregrado que analizaba las relaciones entre rendimiento académico y Mindfulness (Alfaro, 2016), un programa sobre atención plena que se aplicó con estudiantes universitarios (Arrasco, 2018) y una investigación sobre la capacidad de Mindfulness en los docentes de Secundaria (Zorrilla, 2018).

\section{Problemas emocionales del adolescente}

La adolescencia no es la etapa de tormenta y estrés como Hall caracterizaba a principios del siglo pasado, pero si es un momento vital en la que se repiten una serie de problemas. Algunos autores reducen a tres estas problemáticas: las conductas de riesgo, los conflictos con los padres y la inestabilidad emocional (Oliva, 2007) aunque si analizamos los estudios sobre diagnósticos clínicos en población adolescente observamos que los trastornos más prevalentes son el trastorno por déficit de atención e hiperactividad, los problemas de conducta, la ansiedad y la depresión (Zenner, Herrnleben-Kurz \& Walach, 2014). Diversos estudios han mostrado relaciones significativas del rendimiento con ansiedad (García \& De la Peza, 2005), o la depresión y baja autoestima (Ferrel, Vélez \& Ferrel, 2014).

En Perú, las investigaciones sobre los problemas emocionales de los adolescentes, el paso previo para el diseño de políticas que implementen programas de prevención de la salud en niños y adolescentes como recomienda la OMS, son aún reducidas. Según los datos del estudio epidemiológico de salud mental de Lima Metropolitana y Callao, publicado el 2013 en base a encuestas poblacionales, los estados anímicos preexistentes en los adolescentes de estas áreas son negativos. Las encuestas realizadas detectan que los adolescentes informan de irritabilidad $(52,5 \%)$, aburrimiento $(21,2 \%)$ o inquietud $(12,2 \%)$ siempre o casi siempre, entre otras percepciones (Saavedra et al., 2013). También destacamos del estudio que el 25,9\% de la población adolescente consultada informa de ideación suicida en algún momento de su vida. Cuando se justifica el porqué de esa ideación aparecen los problemas con los padres en un 59,2\% de los casos. Aunque existen algunos estudios sobre la problemática de la depresión en población adolescente en el Perú, estos se han realizado con instrumentos no validados para población peruana y por tanto no cumplen con los estándares de publicación propuestos por la American Psychological Association, APA, según un metaanálisis de Navarro-Loli, Moscoso y CalderónDe la Cruz (2017). Se han localizado también trabajos de investigación en población adolescente sobre el auto concepto emocional y su influencia en las conductas antisociales (Palacios-Garay y Coveñas-Lalupú, 2019), la influencia familiar en las conductas antisociales en Arequipa (Ribera y Cahuana, 2016), satisfacción con la vida (Arias, Huamani \& Caycho-Rodríguez, 2018), sobre la ansiedad (García-Zapatero, 1983; Toledo 2006) y se han revisado los trabajos del ilustre 
investigador peruano Ernesto Pollit sobre la desnutrición y el rendimiento académico (Arias, 2016). La revisión que se ha hecho en Dialnet, Redalyc, Scielo y Eric, aunque no sistemática, muestra que el estudio de los problemas emocionales en adolescentes y su relación con el rendimiento académico en Perú aún es un campo poco investigado.

Como correlato de todo lo anteriormente expuesto, podemos afirmar que la educación emocional o el desarrollo de la inteligencia emocional, ambos términos sinónimos (PérezGonzález, 2012) habría de ser uno de los objetivos principales en la planificación de las instituciones educativas. El autor postula que el desarrollo de esta capacidad no solo mejoraría la capacidad del alumno para afrontar los retos de esta difícil etapa en su vida, sino que parece que podría ser un instrumento válido para mejorar su salud mental, a lo largo de toda su vida (Martins, Ramalho \& Morín, 2010). El diseño de esas políticas por parte del Ministerio de Educación debería de estar sustentado por la evidencia sobre los problemas reales de la población adolescente peruana que, como se ha comentado, aún es poca.

\section{Método}

\section{Objetivo e hipótesis}

Después de revisar la literatura científica sobre el tema y exponer algunas de las investigaciones que se han realizado, se planteó una investigación ex post facto correlacional para describir si existen relaciones significativas entre los problemas de los adolescentes, la capacidad de los alumnos de atención al momento presente o Mindfulness y el rendimiento académico en una muestra de alumnos de tercer curso de Secundaria de Trujillo (Perú). En referencia a los problemas de los adolescentes se tuvo en cuenta los indicadores de insatisfacción corporal, ansiedad, depresión, abuso de sustancias, problemas interpersonales, problemas familiares, incertidumbre sobre el futuro, riesgo psicosocial y autoestima y bienestar.

Las hipótesis descriptivas, de asociación, fueron:

H1 Existirán relaciones significativas entre los problemas de los adolescentes auto informados.

H2 Los adolescentes que presenten malestar y problemas clínicos destacados tendrán un promedio de notas académicas inferior que aquellos que no tienen malestar y problemas.

H3 Los adolescentes que tengan mayor capacidad de atención consciente en el momento acreditarán un mayor rendimiento escolar.

H4 Los adolescentes que tengan mayor capacidad de atención consciente presentarán menos problemas clínicos o malestar.

\section{Muestra}

A causa de la limitación de recursos que se disponía para la investigación, se planteó un muestreo no probabilístico, por conveniencia, después de conseguir la implicación de la institución educativa Jorge Basadre Grohmann, en el distrito de Florencia de Mora en Trujillo, La Libertad (Perú) donde se realizó el estudio. La elección de los grupos que participaron en la investigación fue a cargo del centro educativo. La muestra estuvo integrada por dos clases del tercer curso de Secundaria $(\mathrm{N}=76)$. El centro fue elegido en primera opción por oportunidad. La situación de peligrosidad social del distrito de Florencia de Mora de la localidad de Trujillo (Ministerio del Interior, 2016) fue uno de los elementos que contribuyó a la elección del centro. Se hipotetizó, en esta zona de la ciudad una alta presencia de adolescentes con problemas y malestar y un pobre 
rendimiento escolar de los estudiantes. El primer análisis de la muestra mostró que la edad media fue 14,61 ( $\mathrm{SD}=, 697$ y $\mathrm{R}=14-17$ años) y el 55,20\% del alumnado fueron chicos. Destaca que el $51,31 \%$ de los alumnos nacieron durante el segundo trimestre del año. El 85,52 \% de los cuales nacieron en el primer semestre del año y el $14,47 \%$ en el segundo trimestre.

\section{Instrumentos}

Para medir los problemas de los adolescentes se utilizó el Q-PAD (Sica, 2016), un cuestionario adaptado para población latinoamericana con 81 preguntas con cuatro opciones de respuesta (falsa, parcialmente falsa, parcialmente verdadera y verdadera), que permite una administración ágil (aproximadamente se completa en unos 20 minutos). El cuestionario evalúa 8 escalas: insatisfacción corporal, ansiedad, depresión, abuso de sustancias, problemas interpersonales, problemas familiares, incertidumbre sobre el futuro, riesgo psicosocial y autoestima y bienestar. Las puntuaciones se interpretan a través de la web Teacorrige.com y permite extraer los resultados en rangos de percentiles que se distribuyen en cuatro niveles de interpretación: sin significación clínica; precaución; malestar y problemas y malestar y problemas clínicos destacados. La escala de autoestima y bienestar presenta cinco niveles de interpretación: malestar muy destacado; malestar; precaución; zona límite con presencia de ligero malestar; normal; buena autoestima y precaución, excesiva autoestima. El Q-pad cuenta con baremos específicos de México y Colombia y un baremo general de Latinoamérica (Sica, Chiri, Favilli, \& Marchetti, 2016). Los autores informan que se evaluó la fiabilidad en términos de consistencia interna utilizando los coeficientes alfa de Cronbach con un valor promedio de 0,80 y se aplicó un análisis factorial confirmatorio para determinar la bondad del ajuste del momento en la muestra de tipificación de Latinoamérica. Se aplicaron correlaciones policóricas y el estimatorio WLSMV (Weighted Least Squares Mean and Variances Adjusted) con el programa Mplus 6,1 (Muthén \& Muthén, 2010).

Para medir la capacidad de Mindfulness o atención consciente al momento presente se utilizó la adaptación transcultural de la escala CAMM (Child and Adolescent Mindfulness Measure) utilizado para población entre 9 y 18 años. La escala adaptada es un instrumento con 10 preguntas y respuesta tipo Likert (desde nunca $=0$ hasta siempre $=4$ ). Para la interpretación se invierten sus puntuaciones y se obtiene un único factor. El autor de la adaptación informó que la escala cuenta con consistencia interna $(\alpha=0,78)$ y una estructura de un único factor y muestra análisis de validez de criterio $\left(\mathrm{r}_{\mathrm{s}}=0,79\right)$ y convergente $\left(\mathrm{r}_{\mathrm{s}}=0,5\right)$ (Turanzas, 2013).

Para medir el rendimiento escolar se utilizaron las notas académicas de los alumnos participantes. Se recopiló las notas promedio y las notas de cada asignatura: matemáticas; comunicación; inglés; arte; historia, geografía y economía; persona, familia y relaciones humanas; educación física; educación religiosa; ciencia, tecnología y ambiente y educación para el trabajo. Se solicitó las últimas notas que obtuvieron los alumnos antes de la administración de los cuestionarios. Se facilitaron las notas en formato numérico.

\section{Procedimiento de recogida y análisis de datos}

En la primera fase del proyecto se pidió la colaboración a la dirección del centro, los responsables del equipo de dirección pidieron el consentimiento informado a las familias, como había solicitado el investigador para la administración de los cuestionarios y la recogida de las notas escolares, y se procedió a aplicar el proyecto durante el primer trimestre del curso académico 2017-2018 en diversas sesiones. Durante la administración se siguieron las normas de procedimiento del cuestionario Q-PAD y se explicó el funcionamiento del CAMM antes de administrarlo. Se hizo hincapié delante de los alumnos en informar que la información sería tratada estadísticamente y que no se informaría ni al centro ni a los padres sobre el resultado de los cuestionarios de cada alumno. En una tercera fase se procedió a la recopilación de los datos de los cuestionarios, las notas escolares y finalmente al análisis de todo el material numérico 
obtenido con el Statistical Package for the Social Science, SPSS, versión 22 para Windows. En las diferentes sesiones durante la administración, se contabilizaron diversos alumnos que no respondieron los cuestionarios por ausencia o fueron eliminados por dejar ítems en blanco o llenar los cuestionarios manifiestamente mal para ocultar datos. En los resultados aparecen estos datos como perdidos.

Se analizaron los datos con la intención de describir las posibles relaciones que existen entre las variables y conocer si estas relaciones son estadísticamente significativas o fruto del azar y para ello se consideró un nivel de significación del $5 \%(\mathrm{p}<, 05)$. Se procedió a realizar estadísticos descriptivos de los datos en los que se comprobó las medias (M), la desviación típica o estándar (SD), la media del error estándar (SEM) y el intervalo o rango (R) y se aplicó estudio de correlaciones Pearson, con medidas escalares, Spearman, con medidas ordinales, y pruebas $\mathrm{T}$ de Student, para la contrastación de muestras independientes. Para conocer la potencia estadística en las pruebas T de Student $(1-\beta)$ se utilizó el paquete $G^{*}$ Power para garantizar la seguridad de los resultados estadísticos.

\section{Resultados}

\section{Descripción de la muestra y de las notas académicas}

El análisis del rendimiento escolar del primer trimestre de los alumnos mostró que las chicas $(\mathrm{M}=12,76)$ superan a los chicos $(\mathrm{M}=12,31)$ en el promedio de las notas. Se reportó, inicialmente, una relación estadística significativa entre la variable sexo y la asignatura Formación Ciudadana y Cívica, aunque la potencia estadística encontrada, posteriormente, recomendaría reconsiderar esa relación. Se utilizó la prueba T de Student para muestras independientes para contrastarlo. En esta asignatura las chicas puntuan mejor $(M=12,41 \mathrm{SEM}=, 351)$ que los chicos $(\mathrm{M}=11,26 \mathrm{SEM}=$ .279) y la diferencia que muestra el resultado estadístico es significativa $\mathrm{t}(74)=-2,59, \mathrm{p}, 011$, aunque la potencia estadística $(1-\beta=, 55)$ se reporta por debajo de los límites en qué se considera una potencia mínima (Sánchez, Valera, Velandrino \& Marín, 1992). Por otro lado, se observó que existe una relación estadística significativa entre la variable sexo y la asignatura Ciencia, Tecnología y Ambiente. Se utilizó la prueba T de Student para muestras independientes para contrastarlo. Las chicas también puntuan mejor en la asignatura $(\mathrm{M}=12,91 \mathrm{SEM}=, 272)$ que los chicos $(M=11,67 \mathrm{SEM}=.270$ y la diferencia es significativa $\mathrm{t}(74)=-3,22, \mathrm{p}, 011$. En este caso la potencia estadística sí es destacada, con una cifra cercana a $1(1-\beta=, 97)$. En el resto de las asignaturas no se ha observado que las diferencias sean significativas ( $p>, 05)$.

Si analizamos en su totalidad los datos, vemos que las asignaturas en las que el alumnado puntuó peor fueron Comunicación, Matemáticas e Historia, Geografía y Economía. Se resumen los datos en la tabla 1 . 
Tabla 1.

Estadísticos descriptivos de las notas escolares tomada durante el primer trimestre

\begin{tabular}{lrrrrr}
\hline & $\mathrm{N}$ & Mínimo & Máximo & Media & Desv. típ. \\
\hline Matemáticas & 76 & 9 & 16 & 11,24 & 1,591 \\
Comunicación & 76 & 8 & 15 & 11,18 & 1,598 \\
Inglés & 76 & 2 & 17 & 13,36 & 2,134 \\
Arte & 76 & 1 & 17 & 12,37 & 3,111 \\
Historia, Geografía y & 76 & 7 & 13 & 11,29 & 1,422 \\
$\begin{array}{l}\text { Economía } \\
\text { Formación Ciudadana y Cívica }\end{array}$ & 76 & 8 & 17 & 11,78 & 1,991 \\
$\begin{array}{l}\text { Persona Familia y Relaciones } \\
\text { Humanas }\end{array}$ & 76 & 10 & 20 & 13,28 & 1,866 \\
$\begin{array}{l}\text { Educación Física } \\
\text { Educación Religiosa }\end{array}$ & 76 & 10 & 17 & 13,41 & 1,834 \\
Ciencia, Tecnología y & 76 & 0 & 18 & 13,39 & 3,410 \\
Ambiente & 76 & 8 & 15 & 12,22 & 1,778 \\
Educación para el trabajo & 76 & 9 & 18 & 13,38 & 2,160 \\
N válido (según lista) & 76 & & & & \\
\hline
\end{tabular}

El análisis de correlación de Pearson que se realizó muestra que existen diversas relaciones lineales y positivas entre algunas asignaturas, es decir que el alumnado que saca buenas notas en la primera también lo hace en la segunda o viceversa. Destacamos algunas de estas relaciones por su fuerza. Por ejemplo, Matemáticas y Comunicación $\left(\mathrm{r}_{\mathrm{s}}=, 649, \mathrm{p}, 00\right)$, Formación Ciudadana y Cívica y Comunicación $\left(\mathrm{r}_{\mathrm{s}}=, 608, \mathrm{p}, 00\right)$, Persona, Familia y Relaciones Humanas y Comunicación $\left(\mathrm{r}_{\mathrm{s}}=, 586, \mathrm{p}, 00\right)$ o Formación Ciudadana y Cívica y Persona, Familia y Relaciones Humanas $\left(\mathrm{r}_{\mathrm{s}}=, 562, \mathrm{p}, 00\right)$. Las correlaciones no son significativas en todos los casos, en especial cuando se analiza las correlaciones con Educación Física. Esta materia no correlaciona significativamente $(\mathrm{p}>, 05)$ con 5 otras asignaturas.

\section{Resultados de las hipótesis planteadas.}

El análisis del estado de los problemas auto informados de la muestra valida estudiada con el QPAD mostró que: el 25,4\% presenta unos niveles fuera de lo que se consideraría una autoestima normal o buena autoestima, el 11,8\% presenta indicadores de significación clínica en depresión, el $9 \%$ incertidumbre sobre el futuro, el 8,8\% en problemas familiares y riesgo psicosocial, el 7,4\% en abuso de sustancias, el 5,9\% en insatisfacción corporal y el 5,8\% en problemas interpersonales y ansiedad. Para la interpretación de estos porcentajes no se tuvo en cuenta el nivel Precaución, zona límite con presencia de malestar. Se presentan en la tabla 2 y 3 las frecuencias y porcentajes de cada dimensión. 
Tabla 2

Estadísticos descriptivos de los problemas en el adolescente, $Q-P A D$

\begin{tabular}{lrrrrr}
\hline & & & & & $\begin{array}{r}\text { Malestar y } \\
\text { problemas } \\
\text { clínicos }\end{array}$ \\
& $\begin{array}{r}\mathrm{N} \\
\text { (validos) }\end{array}$ & $\mathrm{N}$ & & $\begin{array}{r}\text { Malestar y } \\
\text { problemas }\end{array}$ & $\begin{array}{r}\text { destacados } \\
\text { Insatisfacción corporal }\end{array}$ \\
\hline Ansiedad & 68 & 8 & $5(7,4 \%)$ & $1(1,5 \%)$ & $3(4,4 \%)$ \\
Depresión & 68 & 8 & $8(11,8 \%)$ & $2(2,9 \%)$ & $2(2,9 \%)$ \\
Abuso de sustancias & 68 & 8 & $2(2,9 \%)$ & $4(5,9 \%)$ & $4(5,9 \%)$ \\
Problemas interpersonales & 68 & 8 & $1(1,5 \%)$ & $1(1,5 \%$ & $4(5,9 \%)$ \\
Problemas familiares & 68 & 8 & $4(5,9 \%)$ & $2(2,9 \%)$ & $2(2,9 \%)$ \\
Incertidumbre sobre el & 67 & 9 & $8(8,9 \%)$ & $3(4,4 \%)$ & $3(4,4 \%)$ \\
futuro & 68 & 8 & $4(11,9 \%)$ & $4(6 \%)$ & $2(3 \%)$ \\
Riesgo psicosocial & & & & \\
\hline
\end{tabular}

Nota: Se incluyen solo los datos relativos a precaución y malestar. Se añade el porcentaje válido además de las frecuencias. Para la interpretación se tiene en cuenta solo aquellas frecuencias en que se constata malestar, ya que la dimensión precaución no se considera aún como manifestación clínica.

Tabla 3.

Estadístico descriptivo de las dimensiones de autoestima y bienestar de los alumnos, Q-pad

\begin{tabular}{lrrrrrr}
\hline & & $\mathrm{N}$ & $\mathrm{N}$ & & $\begin{array}{r}\text { Malestar } \\
\text { muy }\end{array}$ & $\begin{array}{r}\text { Precaución. } \\
\text { Excesiva } \\
\text { autoestima }\end{array}$ \\
\hline $\begin{array}{l}\text { Autoestima } \\
\text { y bienestar }\end{array}$ & 67 & 9 & $15(22,4 \%)$ & $6(9 \%)$ & $4(6 \%)$ & $7(10,4 \%)$ \\
(validos) & (perdidos) & Precaución & Malestar & destacado & \\
\hline
\end{tabular}

Nota: Se muestran solo los datos relativos a precaución y malestar. Se añade el porcentaje válido además de las frecuencias. Para la interpretación se tiene en cuenta las frecuencias en que se constata malestar y también la dimensión de excesiva autoestima.

Los datos del cuestionario auto informado mostraron que hay relaciones positivas y significativas entre diferentes problemas de los adolescentes estudiados y correlaciones negativas entre la variable autoestima y bienestar y las variables depresión e insatisfacción. Destaca la variable Abuso de sustancias que solo se relacionó de una forma significativa con el Riesgo psicosocial. Se informan las correlaciones de Spearman $(\rho)$ en la figura 1 y se destacan algunas de ellas $(\rho>, 500)$. Por lo cual podemos afirmar que se cumplió la hipótesis H1. 


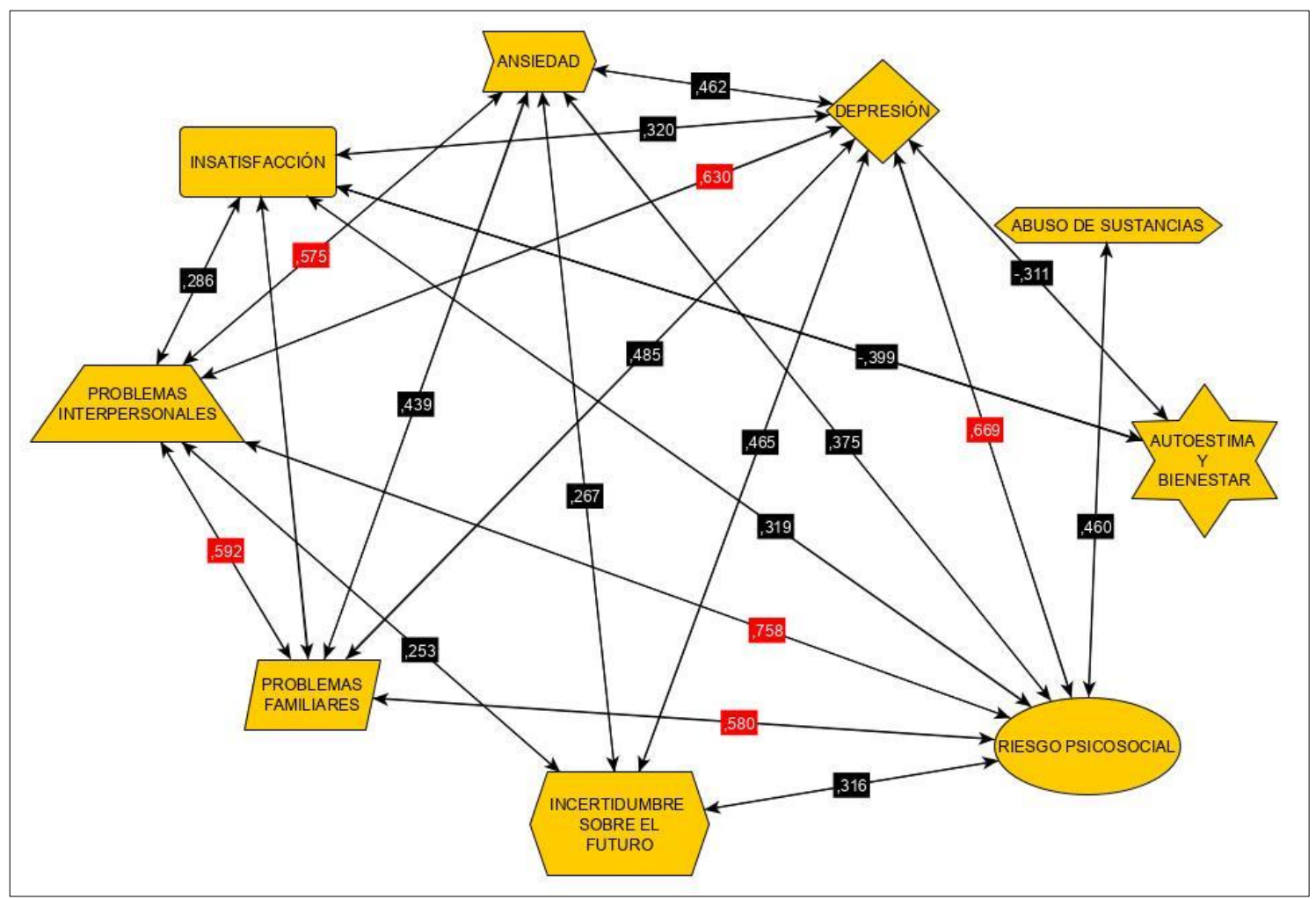

Figura 1: Gráfico de correlaciones de Spearman entre los problemas en el adolescente, auto informados por el alumnado. Se marcan con color rojo algunas de las relaciones $(\rho>, 500)$.

El análisis de los datos de las notas por asignaturas y las diferentes dimensiones de los problemas del adolescente, que presenta el cuestionario auto informado Q-PAD, presentó diversas relaciones, aunque estas fueron bajas. Se observaron unas relaciones significativas, pero de baja intensidad, por tanto, no considerables, entre la incertidumbre sobre el futuro y la asignatura de inglés $(\rho=-, 253, p, 039)$, ansiedad y la asignatura de Historia, Geografía e Economía $(\rho=, 245$, $p, 044)$, depresión y la asignatura de Historia, Geografía e Economía ( $\rho=, 242, p, 046)$, ansiedad y la asignatura Persona, Familia y Relaciones Humanas $(\rho=, 241$, p,048). Si comparamos el promedio de notas con los problemas de adolescente observamos que no existen relaciones significativas en ninguno de las dimensiones analizadas $(\mathrm{p}>, 05)$. Visto los estadísticos realizados, no se cumplió la hipótesis H2.

Cuando observamos los datos de la capacidad del alumno para estar consciente al momento presente, medido a través del CAMM $(\mathrm{M}=33,88 \mathrm{SD}=5,640 \mathrm{R}=23-48)$ y la correlación entre las frecuencias obtenidas con el instrumento y el promedio de las notas académicas pudimos observar que no existe significación estadística $(\rho=, 048, p>, 05)$ por lo cual no se cumplió la hipótesis $\mathrm{H} 3$ planteada.

Finalmente, el análisis de asociación del CAMM con problemas auto informados por el alumnado reportó correlaciones bajas con ansiedad ( $\rho=-, 349, p, 005)$, depresión $(\rho=, 473, p, 000)$, problemas interpersonales $(\rho=-, 309, p, 014)$, incertidumbre sobre el futuro $(\rho=-, 421, p, 001)$ y riesgo psicosocial $(\rho=-, 409, p, 001)$. Aunque estadísticamente los datos son significativos debemos indicar que no se cumple la hipótesis $\mathrm{H} 4$ por considerar que las correlaciones son bajas. 


\section{Discusión}

En relación con las correlaciones entre problemas del adolescente, los datos que se vieron entre las escalas del Q-PAD presentan una dirección semejante con los que se informan en el manual de la adaptación del Q-PAD. La dirección es semejanza en aquellas asociaciones que se presentan más elevadas, pero no en aquellas en que la relación es más débil (Sica et al., 2016). En el manual de la adaptación se describen relaciones bajas en variables que no se han detectado en nuestra muestra. El autor atribuye las diferencias a la muestra utilizada en la adaptación a la lengua española, mayor a la de este estudio.

En esta investigación no aparecieron relaciones significativas entre los problemas de los adolescentes y el promedio de las notas escolares. El autor no pudo comparar esos datos con ningún estudio ya que no se conoce aún ninguna investigación con población adolescente, con este instrumento. El hecho que se describieran relaciones significativas con algunas asignaturas en plantea dudas sobre los resultados, en especial algunas de las direcciones de esa asociación, en sentido contrario a la hipótesis planteada. El autor considera que estas variaciones estarían influenciadas por variables enmascaradas que no se pudieron controlar, como por ejemplo los estilos de enseñanza (Álvarez de Arcaya, 2004), la relación profesor/a -alumno/a, los métodos didácticos utilizados por el profesorado (Martí, 2003) o los enfoques de aprendizaje (Gargallo, Garfela \& Pérez, 2006). Sería deseable, en el futuro, realizar más estudios con muestra peruanas para dilucidar esas dudas.

En este estudio no se detectaron relaciones entre la capacidad de ser consciente al momento presente y el rendimiento escolar. Los datos concuerdan con otro estudio realizado (Rubio y López, 2016) aunque con estudiantes de Bachillerato de España. En otro estudio, en este caso con una muestra de estudiantes de Secundaria, se presentó una relación baja con el promedio de notas, aunque cabe decir que se evaluó los estados de relajación y mindfulness con un cuestionario distinto y por tanto se valoró el constructo con otros ítems (López-González \& Amutio, 2016). En el estudio de Greco, Baer y Smith (2011) se apunta una asociación del CAMM con la competencia académica, aunque se indica en el estudio que fueron débiles. Las diferencias entre esta investigación y la que se presentó aquí está en el hecho que en la investigación de Greco, Baer y Smith considera la dimensión Competencia académica del cuestionario SSRS-TF valorada a partir de la percepción de profesor.

El estudio presentó relaciones significativas entre la capacidad de ser consciente al momento presente y ansiedad, depresión, problemas interpersonales, incertidumbre sobre el futuro y riesgo psicosocial, aunque la intensidad de esas relaciones fue baja. En el estudio antes referido (Greco, Baer, \& Smith, 2011) se relacionan los resultados con síntomas somáticos y problemas externalizantes de comportamiento del adolescente en una dirección semejante a la de esta investigación.

Los resultados de esta investigación deberían ser tomados con cautela. Se realizó un diseño ex post facto correlacional por lo que en ningún caso se podría inferir ninguna generalización a la población adolescente peruana ya que el diseño utilizado permite presentar las covarianzas que existen, pero no presentarlas como fruto de la causalidad, ya que no existe ni aleatorización de la muestra ni tampoco es representativa de la población (Otzen \& Manterola, 2017). Uno de los elementos a tener en cuenta en las limitaciones es el hecho que el Q-PAD presenta un baremo general para Latinoamérica, pero no específicamente para Perú. Esta limitación podría haber afectado los resultados aun mostrando las similitudes que presenta con los resultados del manual de adaptación. Señalamos también que el CAMM ha sido adaptado a Latinoamérica, pero aún no cuenta con baremos específicos del Perú y por tanto se pone en duda su confiabilidad, aunque muestre validez de criterio. 
Se recomienda seguir la investigación con los instrumentos baremados a la población adolescente peruana y ampliar el análisis de los datos que se muestran en la descripción de las notas académicas del alumnado ya que aparecen, por serendipidad, resultados que no se habían tenido en cuenta.

\section{Referencias}

Alfaro, M. (2016). Mindfulness, Flow y rendimiento académico en estudiantes universitarios (Tesis de pregrado). Pontificia Universidad Católica del Perú, Lima.

Álvarez de Arcaya, H. (2004). Influencias de la comunicación no verbal en los estilos de enseñanza y en los estilos de aprendizaje. Revista de Educación, 334, 21-32.

Anderson, P. (1992). Assessment and development of executive function during chilhood. Child Neuropsychology, 8(2), 71-82. doi: http://dx.doi.org/10.1076/chin.8.2.71.8724

Arias, W. (2016). Ernesto Pollitt y su contribución a la psicología evolutiva en el Perú: sus investigaciones en nutrición, cognición y rendimiento escolar. Revista de Psicología, 34(2), 481-500.

Arias, W., Huamani, J., \& Caycho-Rodríguez, T. (2018). Satisfacción con la vida en escolares de la ciudad de Arequipa. Propósitos y Representaciones, 6(1), 351-407. doi: http://dx.doi.org/10.20511/pyr2018.v6n1.206

Ariès, P. (1987). El niño y la vida familiar en el Antiguo Régimen. Madrid: Taurus.

Arrasco, N.E. (2018). Aplicación y Validación del programa Mindfulness para mejorar la atención plena en estudiantes de pregrado de una universidad de Lima, 2017 (tesis de doctorado). Universidad César Vallejo, Lima.

Baer, R. A., Hopkins, J., Krietemeyer, J., Smith, G. T., \& Toney, L. (2006). Using Self-Report Assessment Methods to Explore Facets of Mindfulness. Assessment, 13(1), 27-45. doi: http://dx.doi.org/10.1177/1073191105283504

Bisquerra, R., \& Pérez, N. (2007). Las competencias emocionales. Educación XXI, 10, 61-82. Recuperado de https://www.redalyc.org/pdf/706/70601005.pdf

Blakemore, S.J. (2008). The social brain in adolescence. Nature Reviews. Neuroscience, 9(4), 267-77. doi: http://dx.doi.org/10.1038/nrn2353

Caycho-Rodríguez, Ventura-León, J., Martínez-Munive, R., \& Barboza-Palomino, M. (2019). Traducción y validez de contenido de una escala breve de mindfulness para adolescentes peruanos. Enfermeria Clínica, 29(5), 308-312. doi: http://dx.doi.org/10.1016/j.enfcli.2018.10.002

Caprara, G. V., \& Bonino, S. (E.) (2006). Il comportamento prosociale. Aspetti individuali, familiari e social. Trento: Erickson.

Cohen, J. (1992). A power primer. Psychological Bulletin, 112(1), 155-159.

Damasio, A. (1996). El error de Descartes. Barcelona: Grijalbo-Mondadori

Davies, M., Stankov, L., \& Roberts, R. (1998). Emotional Intelligence. In search of an elusive construct. Journal of Personality and Social Psychology, 7, 989-1015. doi: http://dx.doi.org/10.1037//0022-3514.75.4.989

Diamond, A. (2013). Executive Functions. Annual Review of Psychology, 64, 135-168. doi: http://dx.doi.org/10.1146/annurev-psych-113011-143750

Dictamen $N^{\circ}$ 962. Recomendación del Parlamento Europeo y del Consejo sobre las competencias clave para el aprendizaje permanente. Diario Oficial de la Unión Europa. Bruselas, 18 de diciembre de 2006.

Egocheaga, C.R. (2017). Inteligencia emocional y la convivencia escolar en estudiantes del tercer grado de secundaria de la Institución Educativa Ciro Alegría, Carabayllo-2017 (tesis de grado). Universidad Cesar Vallejo, Lima

Ferrel, F., Vélez, J., \& Ferrel, L. (2014). Factores psicológicos en adolescentes escolarizados con bajo rendimiento académico: depresión y autoestima. Revista Encuentros, 12(2), 35-47 
Flores, J. C., Ostrosky. F., \& Lozano, A. (2012). Batería neuropsicológica de funciones ejecutivas y lóbulos frontales BANFE. México: El Manual Moderno.

García, E., \& De la Peza, R. (2005). Relación de variables cognitivo-emocionales con el rendimiento académico. Iberpsicología, 10(7).

García-Campayo, J., Demarzo, M., \& Modrego, M. (2017). Bienestar emocional y Mindfulness en la educación. Revolucionemos la sociedad del futuro a través de la educación emocional en las escuelas. Madrid: Alianza editorial.

García-Zapatero, G. (1983). Ansiedad debilitadora y rendimiento escolar. Revista De Psicología, 1(1), 61-65. Recuperado de http://revistas.pucp.edu.pe/index.php/psicologia/article/view/3607

Gargallo, B., Garfela, P., \& Pérez, C. (2006). Enfoques de aprendizaje y rendimiento académico en estudiantes universitarios. Bordón, 58(3), 45-61.

Goleman, D. (1995). Inteligencia emocional. Barcelona: Kairós.

Goleman, D. (2006). Inteligencia social. Barcelona: Kairós.

Goldberg, E., \& Costa. L. (1981). Hemisphere differences in the acquisition and use of descriptive systems. Brain Lang,14(1), 144-173. doi: http://dx.doi.org/10.1016/0093934x(81)90072-9

Graber, J.A., \& Brooks-Gunn, J. (1996). Transitions and turning points: Navigating the passage from childhood through adolescence. Developmental Psychology,32, 768-776. doi: http://dx.doi.org/10.1037/0012-1649.32.4.768

Greco, L, Baer, R., \& Smith, G. (2011). Assessing mindfulness in children and adolescents: development and validation of the Child and Adolescent Mindfulness Measure (CAMM). Psycological Assessment, 23(3), 606-614. doi: http://dx.doi.org/10.1037/a0022819

Harvey, P. (2012). An introduction to Buddhism: Teachings, history and practice. Cambridge: Cambridge University Press.

Hayes, S. C., Strosahl, K. D., \& Wilson, K. G. (1999). Acceptance and Commitment Therapy: An experiential approach to behavior change. New York: Guilford Press.

Jha, A. P., Morrison, A. B., Dainer-Best, J., Parker, S., Rostrup, N., \& Stanley, E. A. (2015). Minds "at attention": mindfulness training curbs attentional lapses in military cohorts. PloS one, 10(2). doi: http://dx.doi.org/10.1371/journal.pone.0116889

Kabat-Zinn, J. (2003). Mindfulness-based interventions in context: Past, present, and future. Clinical Psychology: Science and Practice, 10(2), 144-15. doi: http://dx.doi.org/10.1093/clipsy/bpg016

Kuyken, W., Weare, K., Ukoumunne, O. C., Vicary, R., Motton, N., \& Burnett, R. (2013). Efffectiveness of the Mindfulness in Schools Programme; Non-randomised controlled feasibilility study. The British Journal of Psychiatry, 203(2), 126-131. doi: http://dx.doi.org/10.1192/bjp.bp.113.126649

Lewin, K., Lippitt, R., \& White, R. K. (1939). Patterns of aggressive behavior in experimentally created "social climates". The Journal of social psychology, 10(2), 269-299. doi: http://dx.doi.org/10.1080/00224545.1939.9713366

López-González, L., \& Amutio, A. (2016). La competencia en relajación-mindfulness en estudiantes de Educación Secundaria y su influencia en el clima de aula y el rendimiento académico. En M. C. Pérez-Fuentes, J.J. Gázquez, M.C. Molero, Á. Santos, M.C. Simón y A.B. Barragan (Eds.) Variables psicológicas y educativas para la intervención en el ámbito escolar. Volumen II. pp. 301-308. Madrid: Asunivep.

López-González, L., \& Oriol-Granado, X. (2016). La relación entre competencia emocional, clima del aula y rendimiento académico en estudiantes de Secundaria. Cultura y Educación, 28(1), 142-156. doi: http://dx.doi.org/10.1080/11356405.2015.1120448

Lozano, A. (2014). Teoría de teorías sobre la adolescencia. Última década,40, 11-36. doi: http://dx.doi.org/10.4067/S0718-22362014000100002

Martí, E. (2003). Representar el mundo externamente. La construcción infantil de los sistemas externos de representación. Madrid: Antonio Machado. 
Martins, A., Ramalho, N., \& Morín, E. (2010). A comprehensive meta-analysis of the relationship between Emotional Intelligence and health. Personality and Individual differences, 49, 554-564. doi: http://dx.doi.org/10.1016/j.paid.2010.05.029

Mead, M. (1993). Adolescencia, sexo y Cultura en Samoa. Barcelona: Planeta-Agostini.

Ministerio de Educación (2016). Currículo Nacional de la Educación Básica.

Ministerio de Educación (2019). Calificación por letras se implementará en Secundaria de manera gradual. Recuperado de: https://www.gob.pe/institucion/minedu/noticias/25080calificacion-con-letras-se-implementara-en-secundaria-de-manera-gradual

Ministerio de Interior (2016). Ministro de Interior anuncia programa "Barrio Seguro" para Florencia de Mora, El Porvenir y La Esperanza. Recuperado de: https://www.mininter.gob.pe/content/ministro-del-interior-anuncia-programa$\%$ E2\%80\%98barrio-seguro\%E2\%80\%99-para-florencia-de-mora-el-porvenir-y

Mischel. W. (2015). El test de la golosina. Como entender y manejar el autocontrol. Barcelona: Debate.

Moscoso, M., \& Lengacher, C. (2015). Mecanismos neurocognitivos de la terapia basada en mindfulness. Liberabit, 21(1), 221-233.

Muthén, L. K. y Muthén (2010). Mplus: The comprehensive modeling program for applied researchers: User's guide. Los Angeles: Muthén y Muthén.

Navarro-Loli, J., Moscoso, M., \& Calderón-De la Cruz, G. (2017). La investigación de la depresión en adolescentes en el Perú: una revisión sistemática. Liberabit, 23(1), 57-74. doi: http://dx.doi.org/10.24265/liberabit.2017.v23n1.04

Oblitas, L. A., Anicama, J., Calderón, R., Ferrel, R., León, A., \& Liliana, A. (2018). Mindfulness, qigong $\mathrm{y}$ su impacto en la salud. Persona, 21(2). Recuperado de: http://www.redalyc.org/jatsRepo/1471/147158511005/147158511005.pdf

Oliva Delgado, A. (2007). Desarrollo cerebral y asunción de riesgos durante la adolescencia. Apuntes de Psicología, 25(3), 239-254.

OMS (s.f.). Desarrollo en la adolescencia. Recuperado de: https://www.who.int/maternal_child_adolescent/topics/adolescence/dev/es

Otzen, T., \& Manterola, C. (2017). Técnicas de Muestreo sobre una Población a Estudio. International Journal of Morphology,35(1), 227-232. doi: http://dx.doi.org/10.4067/S0717-95022017000100037

Palacios-Garay, J., \& Coveñas-Lalupú, J. (2019). Predominancia del autoconcepto en estudiantes con conductas antisociales del Callao. Propósitos y Representaciones, 7(2), 325-352. doi: http://dx.doi.org/10.20511/pyr2019.v7n2.278

Pérez González, J.C. (2012). Revisión del aprendizaje social y emocional en el mundo. En R. Bisquerra (Ed.), ¿Cómo educar las emociones? La inteligencia emocional en la infancia y la adolescencia, pp. 56-69. Esplugues de Llobregat: Faros Hospital Sant Joan de Déu.

Resolución Ministerial n¹59-2007. Diario Oficial "El Peruano". Lima, 8 de marzo de 2017.

Ribera, R., \& Cahuana, M. (2016). Influencia de la familia sobre las conductas antisociales en Arequipa-Perú. Actualidades en Psicología, 30(12), 85-97. doi: http://dx.doi.org/10.15517/AP.V30I120.18814

Rodríguez-Caycho, T., Ventura, J., Martínez-Munive, R., \& Barboza-Palomino, M. (2018). Traducción y validez de contenido de una escala breve de mindfulness para adolescentes peruanos. Enfermería Clínica. doi: http://dx.doi.org/10.1016/j.enfcli.2018.10.002

Rubio, I., \& López, Y. (2016). Relación entre la capacidad de mindfulness y ansiedad, estrés y rendimiento académico de alumnos de Bachillerato. En J. L. Soler, L. Aparicio, O. Díaz y E. Escolano (Eds). Inteligencia Emocional y Bienestar II: reflexiones, experiencias profesionales e investigaciones. (pp-750-764). Zaragoza: Universidad San Jorge.

Saarni, C. (2000). Emotional Competence. A Developmental Perspective. En R. Bar-On y J. D. A. Parker (Eds.), The Handbook of Emotional Intelligence. Theory, Development, Assessment, and Application at Home, School, and in the Workplace (pp. 68-91). San Francisco: Jossey-Bass. 
Saavedra, J.E., Paz, V., Vargas, H., Robles Y., Pomalina, R., González, S., et al. (2013). Estudio epidemiologico de Salud Mental en Lima Metropolitana y Callao-Replicación 2012. Informe general. Lima: Anales de Salud Mental.

Salovey, P., \& Mayer, J. D. (1990). Emotional intelligence. Imagination, cognition and personality, 9(3), 185-211. doi: http://dx.doi.org/10.2190/DUGG-P24E-52WK-6CDG

Schmeichel, B. J. y Tang, D. (2013). The relationship between individual differences in executive funtioning and emotion regulation: A comprehensive review. En J. Forgas y E. HarmonJones (Eds.). The control within: Motivation and its regulation. (pp.133-52). Nueva York: Psychology Press.

Sica, C., Chiri, L. R., Favilli, R., \& Marchetti, I. (2016). Q-PAD. Cuestionario para la Evaluación de Problemas en Adolescentes (P. Santamaría, adaptador). Madrid: TEA Ediciones.

Siegel, D. J. (2007). The Mindful Brain. Reflection and Attunement in the cultivation of wellbeing. New York: Norton \& Company.

So, K.T., y Orme-Johnson, D. W. (2001). Three randomized experiments on the longitudinal effects of the Transcendental Meditation technique on cognition. Intelligence, 29(5), 419440. doi: http://dx.doi.org/10.1016/s0160-2896(01)00070-8

Soto-Vásquez, M. R., \& Alvarado-García, P. A. A. (2016). Aromaterapia a base de aceite esencial de Peperomia dolabriformis y meditación mindfulness en el tratamiento de la ansiedad. Medicina naturista, 10(1), 47-52.

Southall, D.P., Burr, S., Smith, R.D., Bull, D.N., Radford, A., Williams, A. et al. (2000). The Child-Friendly Healthcare Initiative (CFHI): healthcare provision in accordance with the UN Convention on the Rights of the Child. Pediatrics, 106, 1054-1064. doi: http://dx.doi.org/10.1542/peds.106.5.1054

Spear, L.P. (2007). The psychobiology of adolescence. En K. Kline (Ed.). Authoritative Communities: The Scientific Case for Nurturing Children in Body, Mind, and Spirit. Nueva York: Springer Publishing.

Sporzon, G. (2016). Cerebro ejecutivo, cerebro emocional y neuronas espejo. La aportación de la neurociencia al desarrollo de la Inteligencia Emocional en el currículum escolar. En J.L. Soler, O. Díaz, E. Escolano-Pérez, A. Rodríguez (Eds.). Inteligencia Emocional y Bienestar III. Reflexiones, experiencias profesionales e investigaciones. (pp. 253-263). Zaragoza: Ediciones San Jorge.

Tirapu-Ustárroz, J. y Díaz-Leiva, J. (2018). Las desconexiones interhemisféricas y el papel de la lateralización y diferenciación funcional en los trastornos mentales. Cuadernos de Neuropsicología, 12(1), 42-56.

Toledo, M. (2006). Atención de adolescentes en los Servicios de Salud Mental del hospital Hermilio Valdizán. Avances en salud mental relacional, 5(2). Recuperado de: http://psiqu.com/1-6570

Turanzas, Jorge. (2013). Adaptación Transcultural de la Escala CAMM (Child and Adolescent Mindfulness Measure) y estudio preliminar de sus características psicométricas. doi: http://dx.doi.org/10.13140/RG.2.2.23873.35681

Vallejo, M.A. (2006). Atención plena. EduPsykhé, 5, 231-254-.

Vásquez-Dextre, E. (2016). Mindfulness: Conceptos generales, psicoterapia y aplicaciones clínicas. Revista de Neuropsiquiatría, 79(1), 42-51.

Zanesco, A.P., Denkova, E., Rogers S.L., MacNulty, W. K., \& Jha, A.P. (2018). Mindfulness training as cognitive training in high-demand cohorts: An initial study in elite military servicemembers. Prog Brain Res, 244, 323-354. doi: http://dx.doi.org/10.1016/bs.pbr.2018.10.001

Zelazo, P. D., \& Cunningham, W. A. (2007). Executive function: Mechanims underlying emotion regulation. En J. J. Gross (Ed.), Handbook of Emotion Regulation (pp. 135-158). New York: The Guilford Press.

Zenner, C., Herrnleben-Kurz, S., \& Walach, H, (2014). Mindfulness-based interventions in schools- A systematic review and metaanalysis. Frontiers in Psychology, 5, 603. doi: http://dx.doi.org/10.3389/fpsyg.2014.00603 
Zoogman. S., Goldberg, S.B., Hoyt, W.T. y Miller, L. (2015). Mindfulness interventions with youth: A meta-analysis. Mindfulness, 6(2), 290-302. doi: http://dx.doi.org/10.1007/s12671-013-0260-4

Zorrilla, G. D. (2018). Mindfulness en docentes de las instituciones educativas estatales de mujeres de Chiclayo (tesis de grado). Universidad Cesar Vallejo, Lima. 